University of Nebraska - Lincoln

DigitalCommons@University of Nebraska - Lincoln

$7-2013$

\title{
Predicting the Future of Permanent-Magnet Materials
}

Ralph Skomski

University of Nebraska-Lincoln, rskomski2@unl.edu

Priyanka Manchanda

LMN Institute of Information Technology, priyanka.manchanda@vanderbilt.edu

Pankaj Kumar

Kendriya Vidhyalaya, panksamrat@gmail.com

Balamurugan Balamurugan

University of Nebraska-Lincoln, balamurugan@unl.edu

Arti Kashyap

LMN Institute of Information Technology, akashyap@Inmiit.ac.in

See next page for additional authors

Follow this and additional works at: https://digitalcommons.unl.edu/physicsskomski

Skomski, Ralph; Manchanda, Priyanka; Kumar, Pankaj; Balamurugan, Balamurugan; Kashyap, Arti; and Sellmyer, David J., "Predicting the Future of Permanent-Magnet Materials" (2013). Ralph Skomski Publications. 81.

https://digitalcommons.unl.edu/physicsskomski/81

This Article is brought to you for free and open access by the Research Papers in Physics and Astronomy at DigitalCommons@University of Nebraska - Lincoln. It has been accepted for inclusion in Ralph Skomski Publications by an authorized administrator of DigitalCommons@University of Nebraska - Lincoln. 


\section{Authors}

Ralph Skomski, Priyanka Manchanda, Pankaj Kumar, Balamurugan Balamurugan, Arti Kashyap, and David J. Sellmyer 


\title{
Predicting the Future of Permanent-Magnet Materials
}

\author{
Ralph Skomski ${ }^{1}$, Priyanka Manchanda ${ }^{2}$, Pankaj Kumar ${ }^{2}$, B. Balamurugan ${ }^{1}$, Arti Kashyap ${ }^{2}$, and D. J. Sellmyer ${ }^{1}$ \\ ${ }^{1}$ Physics and Astronomy and NCMN University of Nebraska, Lincoln, NE 68588 USA \\ ${ }^{2}$ School of Basic Sciences, Indian Institute of Technology Mandi, Himachal Pradesh India
}

\begin{abstract}
There are two main thrusts towards new permanent-magnet materials: improving extrinsic properties by nanostructuring and intrinsic properties by atomic structuring. Theory-both numerical and analytical-plays an important role in this ambitious research. Our analysis of aligned hard-soft nanostructures shows that soft-in-hard geometries are better than hard-in-soft geometries and that embedded soft spheres are better than sandwiched soft layers. Concerning the choice of the hard phase, both a high magnetization and a high anisotropy are necessary. As an example of first-principle research, we consider interatomic Mn exchange in MnAl and find strongly ferromagnetic intralayer exchange, in spite of the small Mn-Mn distances.
\end{abstract}

Index Terms-Magnetic anisotropy, magnetization processes, permanent magnets.

\section{INTRODUCTION}

$\mathbf{P}$ ERMANENT magnets [1]-[6] are used in an impressive range of applications, from electromotors and loudspeakers to windscreen wipers, locks, microphones and toy magnets. More recent key developments are applications in computer hard-disk drives, wind generators, and hybrid-car motors. The time of horseshoe magnets has long been over and permanent magnets made from steels are now obsolete, but the high saturation magnetization of $\mathrm{Fe}_{65} \mathrm{Co}_{35}$ and its pronounced temperature stability remain valued in alnico permanent magnets.

The first true hard-magnetic materials, the $L 1_{0}$ compound CoPt [7] and the hexagonal ferrite $\mathrm{BaFe}_{12} \mathrm{O}_{19}$ [8] date back to the mid-20th century, followed by the high-performance rareearth transition-metal permanent magnets Sm-Co [1], [9] and $\mathrm{Nd}_{2} \mathrm{Fe}_{14} \mathrm{~B}$ [2], [10], [11]. This has enabled us to enhance the energy product by two orders of magnitude, from about $1 \mathrm{~kJ} / \mathrm{m}^{3}$ around 1900 to $460 \mathrm{~kJ} / \mathrm{m}^{3}$ in this millennium.

The performance of RE-TM intermetallics is difficult to beat, but rare-earth supplies have become a bottleneck in recent years, and there is active research in various directions. First, as emphasized by Skomski and Coey [12], the range of transitionmetal-rich rare-earth intermetallics is limited, but improving the energy product to about $1000 \mathrm{~kJ} / \mathrm{m}^{3}$ could be possible by suitable nanostructuring [12], [13]. Second, a fundamental topic is the improvement of permanent-magnet materials, by changing chemical composition and atomic structure.

This paper focuses on the theoretical aspects of the first two questions: What are the optimum hard-soft geometries and phases, and what are the prospects for finding new permanent-magnet compounds?

\section{SCIENTIFIC BACKGROUND}

The physics of permanent magnets is based on the distinction between properties of atomic origin (intrinsic properties), and

Manuscript received November 09, 2012; revised January 29, 2013; accepted February 08, 2013. Date of current version July 15, 2013. Corresponding author: R. Skomski (e-mail: rskomski@neb.rr.com).

Color versions of one or more of the figures in this paper are available online at http://ieeexplore.ieee.org.

Digital Object Identifier 10.1109/TMAG.2013.2248139 properties related to the magnets real structure (extrinsic properties), such as coercivity and energy product.

Intrinsic magnetic properties depend on crystal structure and chemical composition. The spontaneous or saturation magnetization $M_{\mathrm{s}}$ and the Curie temperature $T_{\mathrm{c}}$ of most magnets are largely determined by the iron-series (or $3 d$ ) transition-metal (TM) sublattice. Some of the heavier $(4 d, 5 d, 4 f)$ atoms also carry a magnetic moment $m$, but their magnetization $M_{\mathrm{s}}=$ $\mathrm{m} / \mathrm{V}$ is diluted by the larger atomic volume $V$ of the heavy elements. The $3 d$ magnetization is largely determined by the spin $(S)$, which is known as the orbital-moment quenching.

Anisotropy means that the magnetic energy depends on the magnetization directions relative to the crystal axes. Simplifying somewhat, the anisotropic electrostatic crystal field modifies the orbital motion of the electrons and affects, via spin-orbit coupling, the spin system [14]. Since the spin-orbit coupling is a relativistic mechanism, the anisotropy is largest for heavy elements, such as rare earths. Most permanent-magnet alloys have uniaxial (hexagonal, tetrahedral or rhombohedral) crystal structures and the easy magnetization axis ( $c$-axis) perpendicular to the basal plane ( $a$ - $b$-plane) [1]-[3].

Rare-earth moments are unquenched, and the spin-orbit coupling ensures that the $4 \mathrm{f}$ charge clouds, described by Stevens coefficients, are rigidly coupled to the spin [2], [3]. The corresponding rare-earth anisotropy energy is equal to the electrostatic interaction energy between the $4 f$ ions and the anisotropic crystal field.

By contrast, there are no well-defined rules for the $3 d, 4 d$, and $5 d$ anisotropies as a function of the atomic number (or $d$-band filling) $n$. The anisotropy generally oscillates as a function of $n$, but these oscillations involve complicated Fermi-level-dependent $k$-space summations [15]. There are crude rules for nearly filled $3 d$ bands [16], [17], but first-principle approaches, such as VASP calculations are much more reliable. Interestingly, such independent-electron calculations cannot be used to predict rare-earth anisotropy, because $4 f$ electrons are highly correlated and the density functional for rare-earth $4 f$ electrons is not known [18]. Methods such as LSDA $+U$ reproduce the correct configuration or charge state, typically $\mathrm{R}^{3+}$, but become successively less accurate for term, multiplet, and intramultiplet levels. For example, correlations lead to spin-charge separation [18], [19], and even if the charge state is well-known, little can generally be said about the spin direction. 

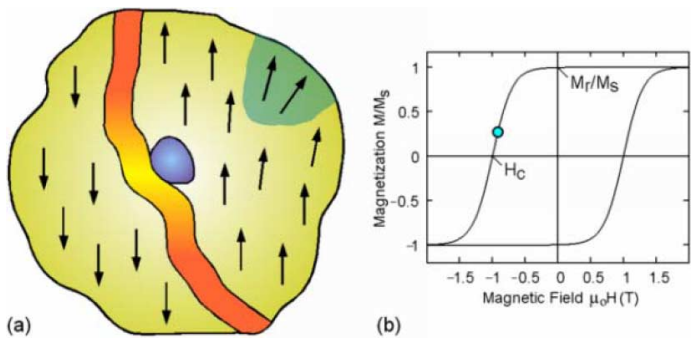

(b)

Fig. 1. Magnetization reversal: (a) nucleation (top right) and pinning (center) as typical reversal mechanisms and (b) hysteresis loop. The circle in (b) roughly corresponds to (a)

It is important to keep in mind that a good permanent magnet needs both a high coercivity and a high magnetization. On a Stoner-Wohlfarth-level, the coercivity $H_{\mathrm{c}}$ is determined by the anisotropy field

$$
H_{\mathrm{A}}=2 K_{1} / \mu_{\mathrm{o}} M_{\mathrm{s}}
$$

The coercivity can therefore be enhanced by reducing the magnetization, and such magnets have been proposed as permanent magnets, most recently a $\mathrm{Rh}$-substituted $\varepsilon-\mathrm{Fe}_{2} \mathrm{O}_{3}$ [20]. However, the energy product is quadratic in the magnetization, and magnets with a low magnetization cannot be used as permanent magnets, even if the coercivity is very high.

Extrinsic properties are usually realized on a scale of several nanometers and on relatively long time-scales, as epitomized by the nonequilibritun character of magnetic hysteresis [21]. There is the general rule that coercivities reach only about $10 \%$ to $30 \%$ of the anisotropy field (Kronmüller factor $\alpha=$ $\left.H_{\mathrm{c}} / H_{\mathrm{A}}\right)$, depending on microstructure. Fig. 1 shows two key mechanisms of coercivity, namely nucleation and pinning. $\mathrm{Nu}-$ cleation-controlled coercivity aims at removing nuclei or "soft spots" and typically implies nearly perfect magnets, without harmful bulk and surface defects. A second mechanism is pinning, where domain walls exist but cannot move due to realstructure imperfections.

Let us consider the nucleation in a soft-phase region and assume that the field $\mathbf{H}=H \mathbf{e}_{\mathrm{z}}$ is parallel to the $c$-axis. Starting from saturation, $\mathbf{M}=M_{\mathrm{s}} \mathbf{e}_{\mathrm{z}}$, nucleation involves a small magnetization component $\mathbf{M}-M_{\mathrm{s}} \mathbf{e}_{\mathrm{z}}=\mathbf{m}$ [21], [22]. The components $m_{\mathrm{x}}$ and $m_{\mathrm{y}}$ of $\mathbf{m}$ are approximately degenerate, so that we can restrict ourselves to the consideration of $m=|\mathbf{m}|[21]$. The analysis starts from the micromagnetic free energy and yields

$$
-\mathrm{A} \nabla^{2} m+\left(K(\mathbf{r})-\mu_{\mathrm{o}} M_{\mathrm{s}} H / 2\right) m=0 .
$$

Here the effective lowest-order uniaxial anisotropy constant $K(\mathbf{r})$ describes the real or defect structure of the magnet and $A$ is the exchange stiffness. $K$ also contains the magnetostatic self-interaction in a rather crude approximation [21]. For homogeneous ellipsoids of revolution, $K(\mathbf{r})=\langle K\rangle$, and (2) reproduces the coherent-rotation result of (1).

\section{Nanostructured Permanent Magnets}

Aligned hard-soft nanocomposites continue to be an active research area [23], [24], [26], [27], in spite of the challenging processing requirements. In these structures, the soft-phase material improves the hard-magnetic performance the main phase, scarifying some anisotropy and coercivity but enhancing magnetization and energy product beyond that of the hard phase [12], [25]. The approach was initially outlined by Kneller and Hawig, who advocated exchange-spring multilayers [13]. Skomski and Coey [12] considered three-dimensional nanostructures and predicted rather accurate upper energy-product limits of about $1000 \mathrm{~kJ} / \mathrm{m}^{3}$. These papers have several precursors, such as earlier work on exchange-spring multilayers [28], [29] and on nucleation fields in inhomogeneous materials [12], [28]-[31]. Actual energy products are always lower than the upper limit, because real-structure imperfections reduce the coercivity [12], [32]. For example, Fig. 2 in [12] shows how the coercivity decreases as the soft regions get bigger. Note that the calculations in [12] and in the present paper assume perfect exchange coupling at the interface. As analyzed in [21], moderately reduced interface exchange has only a very small effect on the interface exchange.

There exist experimental proofs of principle with high magnetization and coercivity in thin films [25], [26], [33]-[36], but the challenge remains to maintain bulk coercivity in the presence of the soft phase. It is well-established that the soft phase of a two-phase system cannot be much larger than twice the Bloch-wall width $\pi \sqrt{A / K_{\mathrm{h}}}$ of the hard phase, irrespective of dimensionality. Otherwise, it was believed that multilayers [13] and spherical inclusions [12], [31] behave similarly, in spite of the more pronounced micromagnetic localization behavior of one-dimensional structures [21]. The specific involvement of $\sqrt{A / K_{\mathrm{h}}}$ is indeed a cornerstone of hard-soft exchange coupling, but there are also differences, both quantitatively and qualitatively.

\section{A. Volume Fraction of the Soft Phase}

How do coercivity and energy product vary as a function of the soft-phase content? In the worst case, a trivially small addition of the soft phase completely ruins the coercivity by creating a harmful nucleus, and the energy product collapses. There are, in fact, thousands of ways to destroy coercivity and energy product. In this subsection, we study two examples of such deterioration mechanisms. By carefully controlling the size and distribution the soft-phase regions, it is possible to limit the coercivity decrease to $H_{\mathrm{c}}=H_{\mathrm{h}}(1-f)$, where $H_{\mathrm{h}}$ is the coercivity of the hard phase and $f$ is the volume fraction of the soft phase. This corresponds to the limit of perfect exchange coupling [12], where $K_{\text {eff }}=\langle K(\mathbf{r})\rangle$. Let us assume that the hysteresis loop is rectangular and the hard-phase coercivity $H_{\mathrm{h}}=\alpha H_{\mathrm{A}}$. The exchange-coupled soft phase enhances the remanence to $M_{\mathrm{r}}=M_{\mathrm{h}}+f\left(M_{\mathrm{s}}-M_{\mathrm{h}}\right)$ but reduces the coercivity. For rectangular loops with $H_{\mathrm{c}}<M_{\mathrm{r}} / 2$, the energy product $(\mathrm{BH})_{\max }=\mu_{\mathrm{o}} H_{\mathrm{c}}\left(M_{\mathrm{r}}-H_{\mathrm{c}}\right)$, whereas for $M_{\mathrm{r}}>H_{\mathrm{c}} / 2,(B H)_{\max }=\mu_{\mathrm{o}} M_{\mathrm{r}}^{2} / 4$. "Poor" hysteresis loops having a straight line from $M\left(-H_{\mathrm{c}}\right)=0$ to $M(0)=M_{\mathrm{r}}$ yield an energy product of $1 / 4 \mu_{\mathrm{o}} M_{\mathrm{r}}^{2} /\left(1+M_{\mathrm{r}} / H_{\mathrm{c}}\right)$. Fig. 2 shows these energy products for different values of $\alpha$.

The maxima in Fig. 2(a) depend on the choice of the hard component and are equal to $1 / 4 \mu_{\mathrm{o}} M_{\mathrm{s}}^{2} /\left(1+\left(M_{\mathrm{s}}-M_{\mathrm{h}}\right) / \alpha H_{\mathrm{A}}\right)$. This means that the ratio $\left(M_{\mathrm{s}}-M_{\mathrm{h}}\right) / \alpha H_{\mathrm{A}} \sim\left(M_{\mathrm{s}}-M_{\mathrm{h}}\right) / K_{\mathrm{h}}$ [35] must be small to achieve high energy products, and there is no point in using semihard materials with relatively small magnetizations and coercivities as hard phases. In fact, the addition of the soft phase deteriorates the energy product if the hard-phase coercivity $\alpha H_{\mathrm{A}}$ is lower than $M_{\mathrm{h}}-M_{\mathrm{s}} / 2$. 


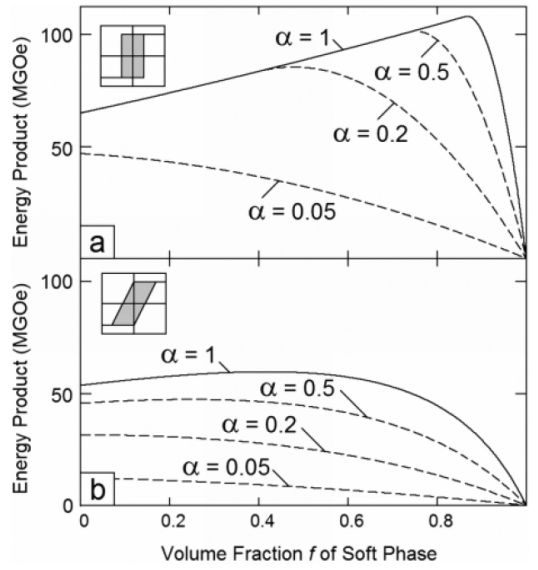

Fig. 2. Energy product of ideally exchange-coupled aligned $\mathrm{Nd}_{2} \mathrm{Fe}_{14} \mathrm{~B}-\mathrm{Fe}$ permanent magnets: (a) rectangular loops and (b) $M(H)$ curves linear in the second quadrant.

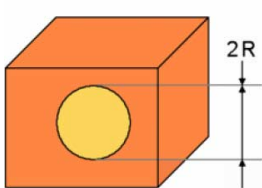

(a)

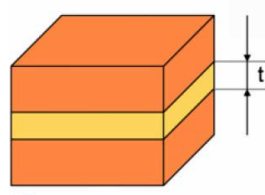

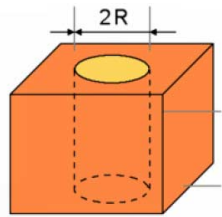

(b)

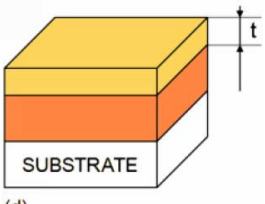

(d)
Fig. 3. Different hard-soft geometries: (a) embedded soft sphere, (b) embedded soft cylinder, (c) soft layer in a hard matrix, and (d) free soft layer at surface. In all cases, the hard matrix is assumed to be $c$-axis aligned, and $t=2 R$ is used in the calculation.

\section{B. Effect of Geometry}

Equation (2) has been solved for a variety of geometries [12], [30], [37]. Fig. 3 summarizes the geometries included in the present analysis, namely spheres, embedded plates, cylinders, and thin films on a hard surface. The nucleation field is obtained by starting from (2) and exploiting the boundary conditions at free and clamped surfaces [12], [31], [37].

Fig. 4 shows the nucleation field as a function of soft-phase dimension (radius or layer thickness), assuming a "proper" exchange length $l_{\mathrm{o}}$ of $\sqrt{A / \mu_{0} M_{\mathrm{s}}^{2}}=2 \mathrm{~nm}, K_{1}=5 \mathrm{MJ} / \mathrm{m}^{2}$ and $\mu_{\mathrm{o}} M_{\mathrm{h}}=1.5 \mathrm{~T}$. For large soft regions $(\mathrm{R} \rightarrow \infty)$, the nucleation mode $m(\mathbf{r})$ is confined to the center of the soft inclusion. The corresponding magnetization profiles are $\phi(z)=\phi_{\mathrm{o}} \cos (\pi z / t)$ for plate-like soft inclusions of thickness $t, \phi_{\mathrm{o}} J_{\mathrm{o}}(r / R)$ for cylindrical inclusions of radius $R\left(J_{\mathrm{o}}\right.$ is a Bessel function), and $2 R \phi_{\mathrm{o}} \sin (\pi r / 2 R) / \pi r$ for spherical inclusions of radius $R$. Explicitly, the ratio $H_{\mathrm{n}} / M_{\mathrm{s}}$ is equal to $19.74 l_{\mathrm{o}}^{2} / R^{2}$ (spheres), $11.57 l_{\mathrm{o}}^{2} / R^{2}$ (cylinders), $19.74 l_{\mathrm{o}}^{2} / t^{2}$ (embedded plates), and $4.94 l_{\mathrm{o}}^{2} / t^{2}$ (soft layer with free surface).

In other words, we see that embedded spheres, Fig. 3(a), have the highest coercivity, corresponding to a factor 4 in the nucleation field compared to embedded layers (c) having $t=2 R$. The behavior of embedded soft cylinders is intermediate between spheres and layers. The nucleation fields of soft-magnetic

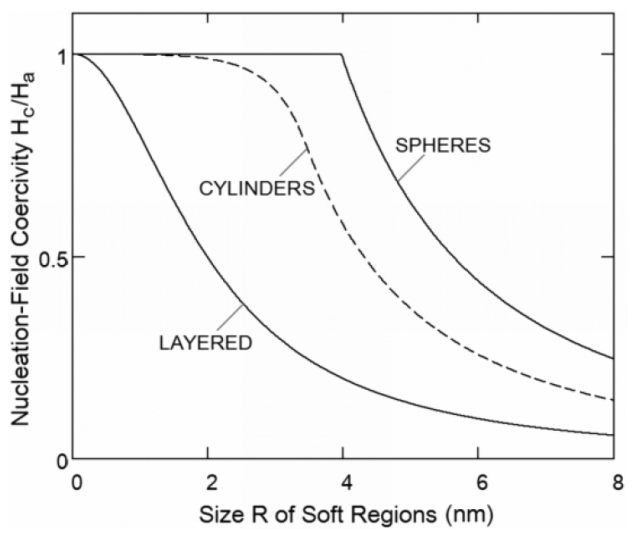

Fig. 4. Schematic soft-phase nucleation field as a function of the radius $R$ of the soft phase. The geometries are those of Fig. 2(a)-(c), and $2 R=t$ for the layered structure.

layers with one free surface, Fig. 3(d), have an additional factor $1 / 4$ compared to the embedded layer, or a factor $1 / 16$ compared to embedded spheres.

For very small soft inclusions, the nucleation field approaches the anisotropy field $H_{\mathrm{a}}$ of the hard phase, but due to localization, the approach to the "plateau" $H_{\mathrm{n}}=H_{\mathrm{a}}$ is dimensionality-dependent. This is in close analogy to the quantum-mechanical delocalization of electrons in an inhomogeneous potential $V(\mathbf{r})$ [21] and to the behavior of impurity states in the band gaps of solids of different dimensionalities [38]. Nucleation modes in one-dimensional systems (layers) localize most easily and exhibit a pronounced nucleation-field drop, whereas three-dimensional (spherical) inclusions need a minimum size to be effective, $R=4 \mathrm{~nm}$ in Fig. 4. Two-dimensional systems (cylinders) form a borderline case, with logarithmically weak localization corrections [38], [39].

\section{Other Micromagnetic Approaches}

Let us consider alnicos, where elongated soft regions of FeCo, Fig. 5(a), are embedded in a nonmagnetic NiAl matrix [4], both phases crystallizing in the cubic $\mathrm{CsCl}$ (B2) structure. The magnetic anisotropy of alnicos is of the shape-anisotropy type, caused by magnetostatic charges at the wires' ends. Since the energy product $(B H)_{\max }$ never exceeds $\mu_{\mathrm{o}} M_{\mathrm{o}}^{2} / 4$, where $M_{\mathrm{o}}=f M_{\mathrm{s}}$, it is advantageous to enhance the volume fraction of the magnetic FeCo phase, the dark area in Fig. 4(a). However, this reduces the coercivity, and the interplay between magnetization increase and coercivity decrease yields a volume fraction $f=2 / 3$ at which the energy product reaches a maximum. For highly idealized alnico, this analysis yield $(B H)_{\max }=\mu_{\mathrm{o}} M_{\mathrm{s}}^{2} / 12[40]$.

What would happen if there was some anisotropy associated with the predominant (001)-type interfaces between the main $(\mathrm{FeCo})$ and matrix $(\mathrm{NiAl})$ phases? Surface and interface anisotropies $K_{\mathrm{s}}$ have values of up to $1 \mathrm{~mJ} / \mathrm{m}^{2}\left[1 \mathrm{erg} / \mathrm{cm}^{2}\right]$. This corresponds to a bulk anisotropy of $5 \mathrm{MJ} / \mathrm{m}^{3}$ (or roughly 0.35 $\mathrm{meV}$ per atom), roughly equal to the maximum anisotropy observed in a $3 \mathrm{~d}$ system $\left(\mathrm{YCO}_{5}\right)$. The physics behind this similarity is the crystal structure of $\mathrm{YCo}_{5}$, which consists of alternating $\mathrm{Co}$ and Y-Co layers, each alternation giving rise to a large 'interface' anisotropy. 


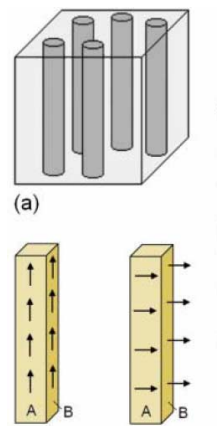

(b)

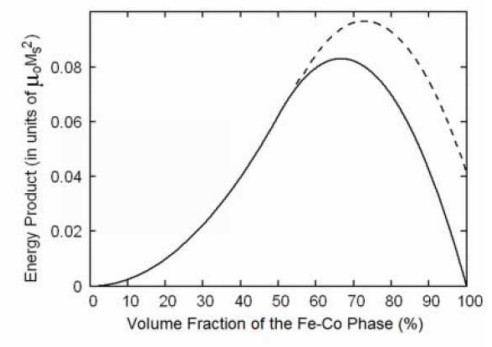

(c)
Fig. 5. Interface effect in alnico: (a) alnico-type structure, (b) schematic spin structure with respect to (001)-type surfaces, and (c) energy product as a function of packing fraction. The solid line in (c) refers to $H_{\mathrm{a}}=0$ [40], whereas the dashed line shows the effect of a positive $H_{\mathrm{a}}$.

An approximate energy product is obtained from (3), by using $H_{\mathrm{c}}=1 / 2(1-f) M_{\mathrm{r}}+H_{\mathrm{a}}$, where $H_{\mathrm{a}}$ is the additional anisotropy-contribution due to the interface anisotropy. If $H_{\mathrm{a}}$ is independent of $f$, then the derivative $\partial H_{\mathrm{c}} / \partial f$ remains unchanged and the energy product is maximized for $H_{\mathrm{c}}=M_{\mathrm{r}} / 4$, irrespective of $H_{\mathrm{a}}$. The optimum packing fraction $f=2\left(1+2 \Delta H_{\mathrm{a}} / M_{\mathrm{s}}\right) / 3$, yields the dashed line in Fig. 5(c).

The net surface anisotropy, obtained by adding the inequivalent surface contributions $\mathrm{A}$ and $\mathrm{B}$ in Fig. 5(b), yields an anisotropy-field change of $\Delta H_{\mathrm{s}}=-4 K_{\mathrm{s}} / \mu_{\mathrm{o}} M_{\mathrm{s}} w$, where $w$ is the width. Negative interface anisotropy, where the easy axis is parallel to the wires, moves the peak in Fig. 5(b) towards higher volume fractions and higher energy-product values. For a long wire having a square cross section $(w=20 \mathrm{~nm})$ and a (001)-type interface, an interface anisotropy of magnitude 1 $\mathrm{mJ} / \mathrm{m}^{2}$ translates into substantial anisotropy-field change of up to $0.1 \mathrm{~T}$ [ $1 \mathrm{kOe}$ ], comparable to the coercivity of alnico. This is important, because the small coercivity is the bottleneck for the use of an otherwise good permanent-magnet material. Note, however, that the change may have either sign, depending the sign of $K_{\mathrm{s}}$.

\section{HARD-MAGNETIC COMPOUNDS}

The search for new intermetallic compounds has been a longstanding task in magnetism, and the rare-earth intermetallics used at present are highly sophisticated and difficult-to-improve alloys. However, exploring new and modified magnetic phases - such as specialty magnets, including thin-film magnets, and rare-earth-free magnets with energy products between alnico and $\mathrm{Nd}-\mathrm{Fe}-\mathrm{B}$ - remains an important research direction and has attracted renewed interest.

This section discusses some first-principle results by the present authors and by other groups. In our own work, we used a supercell approach, the frozen-core full-potential projected augmented wave (PAW) method as implemented in the Vienna ab-initio simulation package (VASP) [41], and a generalized-gradient approximation (GGA) [41], [42].

\section{A. Iron- and Cobalt-Base Magnets}

Iron- and cobalt-based metallic magnets play an important role in magnetism, largely because their magnetization is as high as $2.43 \mathrm{~T}$ in $\mathrm{Fe}_{65} \mathrm{Co}_{35}$. Substantial anisotropy,

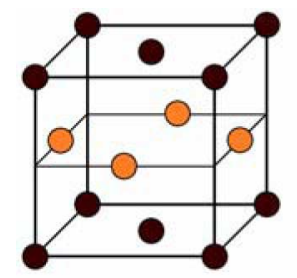

(a)

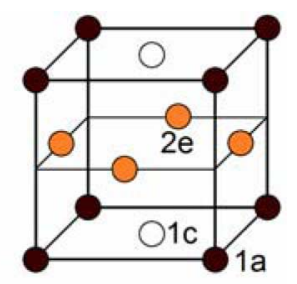

(b)
Fig. 6. $L 1_{0}$ and related structures: (a) fcc and (b) general $L 1_{0}$ structure of the composition $\mathrm{ABC}_{2}$.

$K_{1}=9.5 \mathrm{MJ} / \mathrm{m}^{3}$, and a magnetization of $\mu_{\mathrm{o}} M_{\mathrm{s}}=1.9 \mathrm{~T}$ have been predicted for strongly distorted Fe-Co with $c / a=1.23$ [43]. Experimental room-temperature anisotropies per strained $\mathrm{Fe}$ or $\mathrm{Co}$ atom reach about $2.1 \mathrm{MJ} / \mathrm{m}^{3}$, but this value does not account for the large amount of $\mathrm{Pt}$ (about 75 vol.\%) [44]. In fact, a similar mechanism is responsible for the coercivity of steel magnets, where interstitial carbon yields a tetragonal martensitic lattice distortion [5], and both magnetoelastic and chemical effects contribute to the net anisotropy [45]. Other candidates for nonrare-earth permanent magnets are $\mathrm{Zr}_{2} \mathrm{Co}_{11}$ [46] and orthorhombic $\mathrm{HfCo}_{7}$ [47], which have magnetzations comparable to $\mathrm{Sm}-\mathrm{Co}$, for example $1.09 \mathrm{~T}$ for $\mathrm{HfCo}_{7}$. A problem is that the addition of $4 d$ and $5 d$ atoms, such as $\mathrm{W}$, often deteriorates the magnetization [48].

An interesting noncubic high-magnetization phase is $\mathrm{Fe}_{16} \mathrm{~N}_{2}$ [49], where the nitrogen enhances the magnetization by expanding the Fe lattice. The effect on the electronic structure of the Fe is larger than the dilution of the magnetic moment, although magnetization values of $3.0 \mathrm{~T}$ have not been convincingly verified [49], and the structures are difficult to prepare.

\section{B. $L 1_{0}$-Ordered Magnets}

$L 1_{0}$-ordered alloys such as CoPt, FePt and FePd [7], [5], [50], have long been known in permanent magnetism, but their use has been limited by the price of Pt and Pd. There are a few ferromagnetic Mn alloys with modest magnetizations of less than one tesla, such as MnBi and MnAl [3], [5], [50]. $L 1_{0}$-ordered FeNi (tetrataenite) was originally discovered in meteorites, formed with cooling times in excess of one million years, but is now being explored from the viewpoint of permanent magnetism [51]. Fig. 6 shows the most general $L 1_{0}$ structure, which has the composition $\mathrm{ABC}_{2}$ [52].

In Fe-rich noncubic Pt alloys, a moderate reduction of rawmaterials costs can be achieved by substituting Fe and Co for Pt. For example, recently produced Fe-Co-Pt thin-film magnets have a thickness of $20 \mathrm{~nm}$ and room-temperature properties of up to $\mu_{\mathrm{o}} H_{\mathrm{c}}=2.52 \mathrm{~T}$ and $\mu_{\mathrm{o}} M_{\mathrm{s}}=1.78 \mathrm{~T}$ [26], [35]. In these structures, the compromise between magnetization and coercivity yields a nominal energy product maximum of $510 \mathrm{~kJ} / \mathrm{m}^{3}$ for $\mathrm{Fe}_{40} \mathrm{Co}_{22} \mathrm{Pt}_{38}$.

To understand the magnetic moments in $\mathrm{Fe}_{1-\mathrm{x}} \mathrm{Co}_{\mathrm{x}} \mathrm{Pt}$, we have performed supercell calculations on various chemically ordered and disordered $L 1_{0}$ derivates [42]. The ordered alloys have the nominal composition $\mathrm{Fe}_{8} \mathrm{Co}_{8} \mathrm{Pt}_{16}$ but differ by their stackings, both vertically and laterally. The disordered alloys are approximated by supercells with 32 atoms and have the nominal compositions $\mathrm{Fe}_{15} \mathrm{CoPt}_{16}(\mathrm{x}=6.25 \%)$ and $\mathrm{Fe}_{14} \mathrm{Co}_{2} \mathrm{Pt}_{16}(x=12.5 \%)$. In $\mathrm{Fe}_{15} \mathrm{CoPt}_{16}$, all Co sites are 
equivalent, but in the $\mathrm{Fe}_{14} \mathrm{Co}_{2} \mathrm{Pt}_{16}$ supercell, there are 7 crystallographically nonequivalent configurations - each of these configurations requires a separate first-principle calculation, followed by configurational averaging.

Both our supercell [42] and previous CPA [53] calculations indicate that the average moment decreases with increasing Co content. This is very different from bcc $\mathrm{Fe}_{1-\mathrm{x}} \mathrm{Co}_{\mathrm{x}}$, where the moment initially increases with $x$, from $2.2 \mu_{\mathrm{B}}(\mathrm{Fe})$ to 2.4 $\mu_{\mathrm{B}}\left(\mathrm{Fe}_{65} \mathrm{Co}_{35}\right)$. In $\mathrm{Fe}_{8} \mathrm{Co}_{8} \mathrm{Pt}_{16}$, the effective is $\mathrm{Fe}-\mathrm{Co}$ moment is $2.42 \mu_{\mathrm{B}}$ [42], [54], corresponding to strong 3-D ferromagnetism. Note that perfectly ordered FePt has been predicted to be antiferromagnetic [55]. Our calculations yield a very weak ferromagnetic exchange, $0.005 \mathrm{eV}$ per atom, and the FM-AFM transition is within the margin of error.

\section{Manganese-Based Magnets}

Tripositive manganese has a moment of $5 \mu_{\mathrm{B}}$ per atom, as compared to $2.2 \mu_{\mathrm{B}}$ for $\mathrm{Fe}$. If this moment could be exploited in industrial magnets, it would revolutionize technology far beyond magnetism and open the door for completely new technologies. Furthermore, Mn is a relatively cheap metal, which is of utmost importance in the light of the tight supply and high costs of rare earths. Unfortunately, most manganese compounds are antiferromagnetic, which is typical for elements in the middle of the $3 d$ series. $L 1_{0}$-ordered (or $\tau$-phase) MnAl requires small $\mathrm{C}$ additions to become structurally stable [5], but exhibits appreciable intrinsic properties, namely $\mu_{\mathrm{o}} M_{\mathrm{s}}=0.75$ $\mathrm{T}, K_{1}=1.7 \mathrm{MJ} / \mathrm{m}^{3}$ and $T_{\mathrm{c}}=650 \mathrm{~K}$ [3], [50]. Our focus is on exchange interactions between $\mathrm{Mn}$ atoms, which largely determine the magnetization and Curie temperature of Mn alloys.

The Slater-Bethe-Néel curve suggests that ferromagnetic interatomic exchange in $\mathrm{Mn}$ alloys requires large interatomic distances, with the effect of strongly diluting the magnetization. However, the Slater-Bethe-Néel curve is a crude approximation, and our VASP calculations show that strong ferromagnetic (FM) exchange can exist even for very short Mn-Mn distances. In $L 1_{0}$-ordered $\mathrm{MnAl}$, the dense-packed $\mathrm{Mn}$ sheets exhibit an FM intralayer exchange $J$, whereas the interlayer exchange $J^{\prime}$, with enhanced $\mathrm{Mn}-\mathrm{Mn}$ distances due to the separating $\mathrm{Al}$, is only very weakly ferromagnetic. For the $L 1_{0} \mathrm{MnAl}$ alloys, we obtain $\mathrm{Mn}$ and $\mathrm{Al}$ moments of $2.420 \mu_{\mathrm{B}}$ and $-0.061 \mu_{\mathrm{B}}$, respectively. This means that the coupling between the Mn and $\mathrm{Al}$ sublattices is antiferromagnetic.

It is interesting to see how properties change on Fe addition. Fig. 7 shows the spin-resolved density of states (DOS) for $L 1_{0}$ $\mathrm{MnFeAl}_{2}$, which crystallizes in the structure of Fig. 6(b). The Fe reduces the net moment, with $\mathrm{Fe}, \mathrm{Mn}$ and $\mathrm{Al}$ atomic moments of $2.461 \mu_{\mathrm{B}}, 1.899 \mu_{\mathrm{B}}$, and $-0.056 \mu_{\mathrm{B}}$, respectively. On the other hand, the Fe substitution enhances the anisotropy constant from $1.77 \mathrm{MJ} / \mathrm{m}^{3}$ in $\mathrm{MnAl}$ to $2.5 \mathrm{MJ} / \mathrm{m}^{3}$ in $\mathrm{MnFeAl}_{2}$.

For MnAl, our first-principle calculations yield $J=502 \mathrm{~K}$ and $J^{\prime}=73 \mathrm{~K}$, and an approximate Curie-temperature of 718 $\mathrm{K}$, as compared to the experimental value of about $650 \mathrm{~K}$. For hypothetical $L 1_{0}$-ordered $\mathrm{FeMnAl}_{2}$, the Fe-Mn interlayer coupling is very strong, $J^{\prime}=593 \mathrm{~K}$, but the Mn-Mn intraplane exchange is negative, $J=-185 \mathrm{~K}$, and the spin structure with the lowest energy is antiferromagnetic.

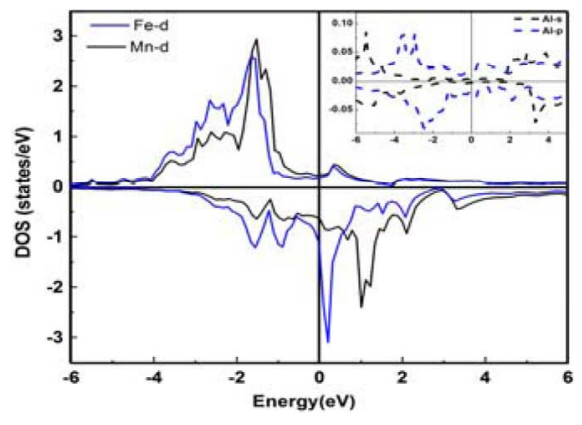

Fig. 7. Spin-polarized majority $(\uparrow)$ and minority $(\downarrow) d$ densities of states (DOS) of $\mathrm{FeMnAl}_{2}$. The inset shows the $s$ and $p$ DOS of Al.

\section{CONCLUSION}

In summary, we have investigated several aspects of $c$-axisaligned hard-soft nanostructures and of permanent-magnet alloys. Concerning the hard-soft geometry, there are two rules. First, soft-in-hard geometries are better than hard-in-soft geometries. Second, among the favorable hard-in-soft geometries, embedded soft spheres are best. Embedded layers are worst, whereas embedded cylinders are intermediate. For small feature sizes, the differences are a micromagnetic analog to the dimensionality-dependent localization behavior of impurity states in the electronic band gaps. The hard-phase materials best suited for hard-soft nanostructuring have both a high magnetization and a high anisotropy.

Our first-principle calculations on MnAl show that a strongly ferromagnetic exchange can exist for very short $\mathrm{Mn}-\mathrm{Mn}$ distances. This is encouraging and calls for future research. Iron addition enhances the anisotropy, from $1.77 \mathrm{MJ} / \mathrm{m}^{3}$ to $2.50 \mathrm{MJ} / \mathrm{m}^{3}$ for $\mathrm{FeMnAl}_{2}$, but reduces the magnetization by creating antiferromagnetic bonds.

So, let us return to our starting point. Can theory really predict the future of permanent magnetism? Probably not. Can it guide experimentalists to go in meaningful directions and to avoid pitfalls? Yes.

\section{ACKNOWLEDGMENT}

This work was supported by ARPA-E REACT (PM, PKS), BREM, and NSF MRSEC DMR-0820521 (RS), DOE (DJS), ARPA-E Delaware (BB), DST (AK), and NCMN. The authors would like to thank B. McCallum, B. Das, T. A. George, H. Jiang, P. Kharel, M. Kramer, L. Lewis, J. E. Shield, W. Zhang, and R. Zhang for discussions related to this paper.

\section{REFERENCES}

[1] K. Kumar, "RETM ${ }_{5}$ and $\mathrm{RE}_{2} \mathrm{TM}_{17}$ permanent magnets development," J. Appl. Phys., vol. 63, pp. R13-57, 1988.

[2] J. F. Herbst, " $\mathrm{R}_{2} \mathrm{Fe}_{14} \mathrm{~B}$ materials: Intrinsic properties and technological aspects," Rev. Mod. Phys., vol. 63, pp. 819-898, 1991.

[3] R. Skomski and J. M. D. Coey, Permanent Magnet.. Bristol, U.K.: Institute of Physics, 1999.

[4] R. A. McCurrie, Ferromagnetic Materials-Structure and Properties. London, U.K.: Academic Press, 1994.

[5] Concise Encyclopedia of Magnetic and Superconducting Materials, J. E. Evetts, Ed. Oxford, U.K.: Pergamon, 1992.

[6] J. M. D. Coey, "Hard magnetic materials: A perspective," IEEE Trans. Magn., vol. 49, no. 12, pp. 4671-4681, Dec. 2011.

[7] W. Jellinghaus, "New alloys with high coercive force," Z. Tech. Physik, vol. 17, pp. 33-36, 1936. 
[8] C. Kooy and U. Enz, "Experimental and theoretical study of the domain contiguration in thin layers of $\mathrm{BaFe}_{12} \mathrm{O}_{19}$," Philips Res. Repts., vol. 15, pp. 7-29, 1960.

[9] K. Strnat, G. Hoffer, J. Olson, W. Ostertag, and J. J. Becker, “A family of new cobalt-base permanent magnet materials," J. Appl. Phys., vol. 38, pp. 1001-1002, 1967

[10] M. Sagawa, S. Fujimura, H. Yamamoto, and Y. Matsuura, "Permanent magnet materials based on the rare earth-iron-boron tetragonal compounds," IEEE Trans. Magn., vol. 20, no. 5, pp. 1584-1589, Sep. 1984.

[11] M. Sagawa, S. Hirosawa, H. Yamamoto, S. Fujimura, and Y. Matsuura, "Nd-Fe-B permanent magnet materials," Japan J. Appl. Phys., vol. 26, pp. 785-800, 1987.

[12] R. Skomski and J. M. D. Coey, "Giant energy product in nanostructured two-phase magnets," Phys. Rev. B, vol. 48, pp. 15812-15816, 1993.

[13] E. F. Kneller and R. Hawig, "The exchange-spring magnet: A new material principle for permanent magnets," IEEE Trans. Magn., vol. 27, no. 4 , pp. $3588-3600$, Jul. 1991.

[14] F. Bloch and G. Gentile, "Zur Anisotropie der Magnetisierung ferromagnetischer Einkristalle," Z. Phys., vol. 70, pp. 395-408, 1931.

[15] R. Skomski, A. Kashyap, A. Solanki, A. Enders, and D. J. Sellmyer, "Magnetic anisotropy in itinerant magnets," J. Appl. Phys., vol. 107, pp. 09A735-1-09A735-3, 2010.

[16] D.-Sh. Wang, R.-Q. Wu, and A. J. Freeman, "First-principles theory of surface magnetocrystalline anisotropy and the diatomic-pair model," Phys. Rev. B, vol. 47, pp. 14932-14947, 1993.

[17] D. Sander, R. Skomski, C. Schmidthals, A. Enders, and J. Kirschner, "Film stress and domain wall pinning in sesquilayer iron films on W(110)," Phys. Rev. Lett., vol. 77, pp. 2566-2569, 1996.

[18] P. Fulde, Electron Correlations in Molecules and Solids. Berlin, Germany: Springer-Verlag, 1991.

[19] A. J. Schofield, "Non-Fermi liquids," Contemporary Phys., vol. 40, pp. 95-115, 1999

[20] A. Namai, M. Yoshikiyo, K. Yamada, Sh. Sakurai, T. Goto, T Yoshida, T. Miyazaki, M. Nakajima, T. Suemoto, H. Tokoro, and Sh.-I. Ohkoshi, "Hard magnetic ferrite with a gigantic coercivity and high frequency millimetre wave rotation," Nature Commun., vol. 3 , pp. 1035-1-1035-6, 2012.

[21] R. Skomski, "Nanomagnetics,” J. Phys.: Condens. Matter, vol. 15, pp. R841-896, 2003

[22] W. F. Brown, Micromagnetics. New York, NY, USA: Wiley, 1963.

[23] N. Jones, "The pull on stronger magnets," Nature, vol. 472, pp. 22-23, 2011.

[24] J. S. Jiang, J. E. Pearson, Z. Y. Liu, B. Kabius, S. Trasobares, D. J. Miller, S. D. Bader, D. R. Lee, D. Haskel, G. Srajer, and J. P. Liu, "Improving exchange-spring nanocomposite permanent magnets," Appl. Phys. Lett, vol. 85, pp. 5293-5295, 2004.

[25] P. Liu, C. P. Luo, Y. Liu, and D. J. Sellmyer, "High energy products. In rapidly annealed nanoscale Fe/Pt multilayers," Appl. Phys. Lett., vol. 72, pp. 483-485, 1998.

[26] Y. Liu, T. A. George, R. Skomski, and D. J. Sellmyer, "Aligned and exchange-coupled FePt-based films," Appl. Phys. Lett., vol. 99, pp. 172504-1-172504-3, 2011.

[27] W.-B. Cui, Y. K. Takahashi, and K. Hono, " $\mathrm{Nd}_{2} \mathrm{Fe}_{14} \mathrm{~B} / \mathrm{FeCo}$ Anisotropic nanocomposite films with a large maximum energy product," Adv. Mater., vol. 24, pp. 6530-6535, 2012.

[28] E. Goto, N. Hayashi, T. Miyashita, and K. Nakagawa, "Magnetization and switching characteristics of composite thin magnetic films," J. Appl. Phys., vol. 36, pp. 2951-2958, 1965.

[29] S. Nieber and H. Kronmüller, "Nucleation fields in periodic multilayers," Phys. Stat. Sol. (b), vol. 153, pp. 367-375, 1989.

[30] H. Kronmüller, "Theory of nucleation fields in inhomogeneous ferromagnets," Phys. Stat. Sol. (b), vol. 144, pp. 385-396, 1987.

[31] R. Skomski, "Nucleation in inhomogeneous permanent magnets," Phys. Stat. Sol. (b), vol. 174, pp. K77-80, 1992.

[32] R. Skomski, "Aligned two-phase magnets: Permanent magnetism of the future?," J. Appl. Phys., vol. 76, pp. 7059-7064, 1994.

[33] J. Zhang, Y. K. Takahashi, R. Gopalan, and K. Hono, "Sm(Co, $\mathrm{Cu})_{5} / \mathrm{Fe}$ exchange spring multilayer films with high energy product,' Appl. Phys. Lett., vol. 86, pp. 122509-1-122509-2, 2005.

[34] D. Roy and P. S. A. Kumar, "Enhancement of $(\mathrm{BH})_{\max }$ in a hard-soft-ferrite nanocomposite using exchange spring mechanism," J. Appl. Phys., vol. 106, pp. 073902-1-073902-4, 2009.
[35] P. K. Sahota, Y. Liu, R. Skomski, P. Manchanda, R. Zhang, H. Fangohr, M. Franchin, G. C. Hadjipanayis, A. Kashyap, and D. J. Sellmyer, "Ultrahard magnetic nanostructures," J. Appl. Phys., vol. 111, pp. 07E345-1-07E345-3, 2012.

[36] V. Neu, S. Sawatzki, M. Kopte, C. Mickel, and L. Schultz, "Fully epitaxial, exchange coupled $\mathrm{SmCo} / \mathrm{Fe}$ multilayers with energy densities above 400 kJ/m," IEEE Trans. Magn., vol. 48, no. 11, pp. 3599-3602, Nov. 2012.

[37] R. Skomski, J.-P. Liu, and D. J. Sellmyer, "Quasicoherent nucleation mode in two-phase nanomagnets," Phys. Rev. B, vol. 60, pp. 7359-7365, 1999

[38] E. N. Economou, Green's Functions in Quantum Physics. Berlin Springer, 1979.

[39] R. Skomski, Simple Models of Magnetism. Oxford: University Press, 2008.

[40] R. Skomski, Y. Liu, J. E. Shield, G. C. Hadjipanayis, and D. J. Sellmyer, "Permanent magnetism of dense-packed nanostructures," $J$ Appl. Phys., vol. 107, pp. 09A739-1-09A739-3, 2010.

[41] G. Kresse and J. Hafner, "Ab initio molecular dynamics for open-shell transition metals," Phys. Rev. B, vol. 48, pp. 13115-13118, 1993.

[42] P. Manchanda, R. Skomski, J. E. Shield, S. Constantinides, and A Kashyap, "Intrinsic magnetic properties of $L 1_{0}$-based $\mathrm{Mn}-\mathrm{Al}$ and Fe-Co-Pt alloys," in Proc. REPM'12, Nagasaki, 2012, pp. 115-118.

[43] T. Burkert, L. Nordström, O. Eriksson, and O. Heinonen, "Giant magnetic anisotropy in tetragonal FeCo alloys," Phys. Rev. Lett., vol. 93, pp. 027203-1-027203-4, 2004.

[44] G. Andersson, T. Burkert, P. Warnicke, M. Björck, B. Sanyal, C. Chacon, C. Zlotea, L. Nordström, P. Nordblad, and O. Eriksson, "Perpendicular magnetocrystalline anisotropy in tetragonally distorted Fe-Co alloys," Phys. Rev. Lett., vol. 96, pp. 037205-1-037205-4, 2006.

[45] R. Skomski, V. Sharma, B. Balamurugan, J. E. Shield, A. Kashyap, and D. J. Sellmyer, S. Kobe and P. McGuinness, Eds., "Anisotropy of doped transition-metal magnets," in Proc. REPM'10, Ljubljana, 2010 , pp. 55-60, Jozef Stefan Institute.

[46] B. Das, B. Balamurugan, W. Y. Zhang, R. Skomski, E. S. Krage, S. R. Valloppilly, J. E. Shield, and D. J. Sellmyer, "Magnetism of less common cobalt-rich alloys," in Proc. REPM'12, Nagasaki, 2012, pp. 427-430.

[47] B. Balamurugan, B. Das, V. R. Shah, R. Skomski, X. Z. Li, and D. J. Sellmyer, "Assembly of uniaxially aligned rare-earth-free nanomagnets," Appl. Phys. Lett., vol. 101, pp. 122407-1-122407-5, 2012.

[48] F. Golkar, M. J. Kramer, Y. Zhang, R. W. McCallum, R. Skomski, D. J. Sellmyer, and J. E. Shield, "Structure and magnetic properties of Co-W clusters produced by inert gas condensation," J. Appl. Phys., vol. 111 pp. 07B524-1-07B524-3, 2012

[49] J. M. D. Coey, K. O’Donnell, Q. Qinian, E. Touchais, and K. H. Jack, "The magnetization of $\alpha^{\prime \prime} \mathrm{Fe}_{16} \mathrm{~N}_{2}$," J. Phys.: Condens. Matter, vol. 6, pp. L23-L28, 1994.

[50] T. Klemmer, D. Hoydick, H. Okumura, B. Zhang, and W. A. Soffa "Magnetic hardening and coercivity in $L 1_{0}$ ordered FePd ferromagnets," Scripta Met. Mater., vol. 33, pp. 1793-1805, 1995.

[51] L. H. Lewis, K. Barmak, J. G. Goldstein, F. Pinkerton, and R. Skomski, "Towards stabilization of $L 1_{0}$-type FeNi compounds for permanent magnet applications," in Proc. REPM'12, Nagasaki, 2012 , pp. $102-105$.

[52] R. Skomski, "Phase formation in $L 1_{0}$ magnets," J. Appl. Phys, vol. 101, pp. 09N517-1-09N517-3, 2007.

[53] M. E. McHenry, B. Ramalingum, S. Willoughby, J. MacLaren, and S. G. Sankar, "First principles calculations of the electronic structure of $\mathrm{Fe}_{1-\mathrm{x}} \mathrm{Co}_{\mathrm{x}}$ Pt," IEEE Trans. Magn., vol. 37, no. 4, pp. 1277-1279, Jul. 2001.

[54] A. Kashyap, R. Skomski, A. K. Solanki, Y. F. Xu, and D. J. Sellmyer, "Magnetism of $\mathrm{L} 1_{0}$ Compounds with the Composition MT $(M=\mathrm{Rh}$, Pd, Pt, Ir and $T=$ Mn, Fe, Co, Ni)," J. Appl. Phys., vol. 95, pp. 7480-7482, 2004

[55] G. Brown, B. Kraczek, A. Janotti, T. C. Schulthess, G. M. Stocks, and D. D. Johnson, "Competition between ferromagnetism and antiferromagnetism in FePt," Phys. Rev. B, vol. 68, pp. 052405-1-052405-4, 2003. 